\title{
Carbon Nanoparticle-Enhanced Immunoelectrochemical Detection for Protein Tumor Marker with Cadmium Sulfide Biotracers
}

\author{
Ja-an Annie Ho, ${ }^{*},{ }^{\dagger}$ Yeh-Chun Lin, ${ }^{\dagger}$ Li-Sheng Wang, ${ }^{\dagger}$ Kuo-Chu Hwang, ${ }^{\ddagger}$ and Pi-Tai Chou ${ }^{\mathcal{8}}$ \\ BioAnalytical Chemistry Laboratory, Department of Chemistry, National Tsing Hua University, Hsinchu, 30013 Taiwan, \\ and Department of Chemistry, National Taiwan University, Taipei, 10617 Taiwan
}

We have developed a sensitive electrochemical immunoassay system for the detection of a protein tumor marker, carcinoembryonic antigen (CEA), that is based on a carbon nanoparticle (CNP)/poly(ethylene imine) (PEI)-modified screen-printed graphite electrode (CNPPEI/SPGE) covered with anti-CEA antibodies. The signal amplification strategy-using CdS nanocrystals as biotracers and CNPs to enhance electron transfer-improves the sensitivity and detection limit for CEA, suggesting that this system holds promise for development into a point-of-care or disposable home-care self-diagnostic tool. This biosensor is based on a sandwich complex immunoassay, which we assembled from sequential layers of the anti-CEA antibody ( $\alpha \mathrm{CEA}$ ) on CNP-PEI/SPGE, the CEA sample, and the CdS nanocrystal quantum dots (QDs) sensitized with $\alpha \mathrm{CEA}$ ( $\alpha \mathrm{CEA}-\mathrm{CdS} \mathrm{QD})$. We used square wave anodic stripping voltammetry (SWASV) to amplify the signal current response obtained from the dissolved $\alpha \mathrm{CEA}-\mathrm{CdS}$ QDs. The calibration curve for CEA concentration was linear in the range of $0.032-10 \mathrm{ng} / \mathrm{mL}$; the detection limit (estimated as the mean of the blank sample plus three times the standard deviation obtained on the blank sample) was $32 \mathrm{pg} / \mathrm{mL}$ (equivalent to $160 \mathrm{fg}$ in a 5 $\mu \mathrm{L}$ sample). This method is suitably precise and sensitive to function as a means of determining urinary CEA, which is a better marker than serum CEA for the early detection of urothelial carcinoma.

The detection of trace amounts of target proteins in biological matrixes remains a great challenge in clinical diagnosis, biomedical research, environmental analysis, and food quality control. Early detection of tumor markers is a vital aspect of clinical research, early diagnosis, differentiating diagnosis, curative effect monitoring, and follow-up examinations of patients with tumors or carcinomas. ${ }^{1}$ An ideal tumor marker would clearly distinguish between normal and diseased states with high sensitivity and specificity. The levels of the tumor markers in human serum can

* To whom correspondence should be addressed. Fax: +886-3-571-1082. E-mail: jaho@mx.nthu.edu.tw.

${ }^{\dagger}$ BioAnalytical Chemistry Laboratory, Department of Chemistry, National Tsing Hua University.

* Department of Chemistry, National Tsing Hua University.

$\$$ Department of Chemistry, National Taiwan University.

(1) Faraggi, D.; Kramar, A. Urol. Oncol. 2000, 5, 211-213. provide useful information regarding the stage of the disease, the grade of metastasis, and the recidivism of the tumor. ${ }^{2}$

Carcinoembryonic antigen (CEA), a glycoprotein having a molecular mass of ca. $200 \mathrm{kDa}$, is found in many types of cells associated with tumors; it is one of the most widely used tumor makers for clinical diagnosis. CEA is associated with colon cancer, ${ }^{1,3}$ lung cancer, ${ }^{4}$ urothelialcarcinoma,${ }^{5}$ ovarian carcinoma, ${ }^{6}$ and breast cancer. ${ }^{7}$ Because the CEA levels in sera and other biofluids are related to the state of the tumor, it can be used directly as a marker to evaluate curative effects or to judge recrudescence or metastasis. Monitoring the level of urinary CEA is particularly helpful when evaluating urothelial dysplasia ${ }^{8}$ and in patients with bilharzial chronic cystitis; ${ }^{9}$ therefore, it may be adapted as a valuable screening test for premalignant lesions and malignant transformations. ${ }^{10}$ According to Fraser et al., ${ }^{11}$ the level of urinary CEA increased in $61 \%$ of patients with transitional cell carcinomas of the bladder. Several methods and strategies have been reported for the determination of CEA, including the use of radioimmunoassays, ${ }^{12}$ enzyme immunoassays,${ }^{13}$ fluoroimmunoas-

(2) Szilvás, Á.; Blázovics, A.; Székely, G.; Dinya, E.; Fehér, J.; Mózsik, G. J. Physiol.(Paris) 2001, 95, 247-252.

(3) (a) Schlageter, M. H.; Larghero, J.; Cassinat, B.; Toubert, M. E.; Borschneck, C.; Rain, J. D. Clin. Chem. 1998, 44, 1995-1998. (b) Duffy, M. J.; van Dalen, A.; Haglund, C.; Hansson, L.; Klapdor, R.; Lamerz, R.; Nilsson, O.; Sturgeon, C.; Topolcan, O. Eur. J. Cancer 2003, 39, 718-727.

(4) (a) Kleisbauer, J.; Castelnau, O.; Thomas, P.; Ramirez, J.; Lanteaume, A.; Roux, F. Lung Cancer 1996, 15, 148-148(b) Hernandez, L.; Espasa, A.; Fernandez, C.; Candela, A.; Martin, C.; Romero, S. Lung Cancer 2002, 36, 83-89.

(5) Hall, R. R.; Laurence, D. J. R.; Darcy, D.; Stevens, U.; James, R.; Roberts, S.; Neville, A. M. Br. Med. J. 1972, 3, 609-611.

(6) Engelen, M. J. A.; de Bruijn, H. W. A.; Hollema, H.; ten Koor, K. A.; Willemse, P. H. B.; Aalders, J. G.; van der Zee, A. G. J. Gynecol. Oncol. 2000, 78, 16-20.

(7) (a) Bremer, K.; Micus, S.; Bremer, G. Eur. J. Cancer 1995, 31A, 12551255(b) Jezersek, B.; Cervek, J.; Rudolf, Z.; Novakovic, S. Cancer Lett. 1996, 110, 137-144. (c) Sahin, B.; Paydak, V.; Paydas, S. Eur. J. Cancer 1996, 32 , S24-S24.

(8) Murphy, W. M.; Vandevoorde, J. P.; Rao, M. K.; Soloway, M. S. J. Urol. 1977, 118, 806-808.

(9) Saied, G. M.; El-Metenawy, W. H.; Elwan, M. S.; Dessouki, N. R. World J. Surg. Oncol. 2007, 5, 4.

(10) (a) El-Metenawy, W. H. J. Egypt. Natl. Cancer Inst. 1987, 3, 195-204. (b) Alsabti, E. A.; Kamel, A. Neoplasma 1979, 26, 603-609.

(11) Fraser, R. A.; Ravry, M. J.; Segura, J. W.; Go, V. L. J. Urol. 1975, 114, 226-229.

(12) (a) Szturmowicz, M.; Tomkowski, W.; Fijalkowska, A.; Sakowicz, A.; Filipecki, S. Eur. J. Cancer 1995, 31, S264-S264. (b) Villena, V.; EchaveSustaeta, J.; Martin-Escribano, P.; Ortutio-de-Solo, B. Cancer 1996, 78, 736740. (c) Behera, D.; Raghavendra, V.; Khanduja, K. L. Lung Cancer 1997, $18,168-168$.

10.1021/ac801832h CCC: $\$ 40.75 \odot 2009$ American Chemical Society Published on Web 01/21/2009 
says, ${ }^{14}$ and piezoelectric immunosensors. ${ }^{15}$ Nevertheless, each of these methods suffers from drawbacks: radiation hazards, long analysis times, or the need for qualified personnel and/or sophisticated instrumentation. ${ }^{16}$ Thus, alternative approaches toward CEA determination remain desirable. Electrochemical biosensors offer several advantages relative to the previously developed molecular detection approaches, including the ability to analyze complicated body fluids, high sensitivity, compatibility with microfabrication technologies, low manpower requirements, and compact instrumentation that is compatible with portable devices. ${ }^{17}$ Electrochemical immunosensors based on screenprinted graphite electrodes (SPGE) can be competitive with conventional electrochemical biosensors because of their easy fabrication, disposability, and portability. ${ }^{18}$

Carbon nanomaterials have attracted considerable attention recently because their extraordinary physical properties allow them to be used for the preparation of nanomaterial-reinforced composites, which are widely used in electroanalysis. ${ }^{19}$ The benefits of nanocarbons and fullerenes have triggered new developments in the design of modified electrodes for electrochemical sensing. ${ }^{20}$ Carbon nanotube (CNT) composites with Teflon, epoxy, ${ }^{21}$ chitosan, ${ }^{22}$ polypyrrole, ${ }^{23}$ and poly (ethylenedioxythiophene $)^{24}$ exhibit improved robustness and facilitate immobilization of biocomponents. Composites formed through the coupling of polymers with CNTs and biorecognition elements are becoming increasingly useful because they are simple to construct and, more importantly, they can function as electrochemical biosensors when conducting materials are incorporated into their porous polymers. The remarkable conductivities of multiwalled (MW) CNTs and their appealing electrochemical properties have led recently to an intense amount of research toward the development of CNT-based electrochemical sensors. Most CNTmodified electrodes have been prepared by casting CNT films onto the surfaces of glassy carbon electrodes. Although this approach is useful for studying the electrocatalytic properties of CNTs, new fabrication schemes and other materials are required if we are to produce more rigid and conductive composites and

(13) (a) Delarosa, A. M.; Kumakura, M. Anal. Chim. Acta 1995, 312, 85-94. (b) Pina, T. C.; Zapata, I. T.; Hernandez, F. C.; Lopez, J. B.; Paricio, P. P.; Hernandez, P. M. Clin. Chim. Acta 2001, 305, 27-34. (c) Tang, D. P.; Yuan, R.; Chal, Y. Q. Anal. Chem. 2008, 80, 1582-1588.

(14) Yuan, J. L.; Wang, G. L.; Majima, K.; Matsumoto, K. Anal. Chem. 2001, 73, 1869-1876.

(15) Zhang, B.; Zhang, X.; Yan, H.-h.; Xu, S.-j.; Tang, D.-h.; Fu, W.-l. Biosens. Bioelectron. 2007, 23, 19-25.

(16) Darain, F.; Park, S. U.; Shim, Y. B. Biosens. Bioelectron. 2003, 18, 773780 .

(17) Merkoçi, A.; Aldavert, M.; Marín, S.; Alegret, S. Trends Anal. Chem. 2005, 24, 341-349.

(18) (a) Valat, C.; Limoges, B.; Huet, D.; Romette, J. L. Anal. Chim. Acta 2000, 404, 187-194. (b) Gao, Q.; Ma, Y.; Cheng, Z. L.; Wang, W. D.; Yang, M. R. Anal. Chim. Acta 2003, 488, 61-70. (c) Wu, J.; Tang, J.; Dai, Z.; Yan, F.; Ju, H.; Murr, N. E. Biosens. Bioelectron. 2006, 22, 102-108.

(19) Wildgoose, G. G.; Banks, C. E.; Leventis, H. C.; Compton, R. G. Microchim. Acta 2006, 152, 187-214.

(20) Banks, C. E.; Compton, R. G. Anal. Sci. 2005, 21, 1263-1268.

(21) (a) Pumera, M.; Merkoci, A.; Alegret, S. Sens. Actuators, B 2006, 113, 617-622. (b) Perez, B.; Pumera, M.; del Valle, M.; Merkoci, A.; Alegret, S. J. Nanosci. Nanotechnol. 2005, 5, 1694-1698.

(22) (a) Zhang, M.; Smith, A.; Gorski, W. Anal. Chem. 2004, 76, 5045-5050. (b) Jiang, L.; Wang, R.; Li, X.; Jiang, L.; Lu, G. Electrochem. Commun. 2005 , 7, 597-601.

(23) Wang, J.; Musameh, M. Anal. Chim. Acta 2005, 539, 209-213.

(24) Viswanathan, S.; Wu, L. c.; Huang, M. R.; Ho, J. a. A. Anal. Chem. 2006, $78,1115-1121$. broaden the range of applications of CNT-based electrochemical sensors. ${ }^{24}$ Carbon nanoparticles (CNPs) also display unique advantages over macroelectrodes when used in electroanalysis applications, including enhanced mass transport and catalysis, highly effective surface areas, and control over the electrode microenvironment. CNPs are similar to metal nanoparticles ${ }^{25}$ in that they are high-surface-area building blocks for thin-film electrode systems and because their high contents of interfacial edge sites are potentially beneficial for electrochemical processes. Surface-functionalized CNPs have been investigated for their use in such applications as antioxidant and stabilizer adsorption. ${ }^{26}$ Metal NPs have also been used in a variety of bioanalytical formats with electrochemical detection. Quantum dots (QD), such as CdS nanocrystals, have been employed as electroactive tags for electrochemical assays of cancer marker and DNA. ${ }^{27}$ When NPs are used as quantitation tags, the electrochemical signal emanating from them can then be quantified. ${ }^{28}$ Although several new NPs have been studied extensively for the advanced electrochemical detection of proteins, ${ }^{29}$ the combination of CNPs and $\mathrm{CdS}$ nanocrystals as electrode modifiers and signal amplifiers, respectively, for the electrochemical detection of the cancer marker CEA has not yet been reported (CNPs along with PEI were used in this study to modify the screen-printed electrodes; CdS QDs were used as signal amplifier to detect CEA). This paper presents a novel sensing platform for the determination of trace levels of CEA in standard and human urine samples; this system, which is based on an anti-CEA antibody-immobilized CNP/poly (ethylene imine) (PEI)-modified SPGE ( $\alpha \mathrm{CEA}-\mathrm{CNP}-\mathrm{PEI} / \mathrm{SPGE})$ and CdS nanocrystals labeled with anti-CEA antibodies ( $\alpha$ CEA-CdS QD), can be further developed for in-home care self-diagnostics.

\section{EXPERIMENTAL METHODS}

Reagents. CEA (from human fluids), sodium sulfide nonahydride, 11-mercaptoundecanoic acid (MUA), potassium chloride, sodium chloride, potassium phosphate monobasic $\left(\mathrm{KH}_{2} \mathrm{PO}_{4}\right)$, potassium phosphate dibasic $\left(\mathrm{K}_{2} \mathrm{HPO}_{4}\right)$, sodium acetate, mercuric nitrate monohydrate, and 50\% PEI were purchased from Sigma-Aldrich Chemical Co. (St. Louis, MO). Polyclonal anticarcinoembryonic antigen antibody $(\alpha \mathrm{CEA})$ was obtained from Biocare Medical (Concord, CA). 1-Ethyl-3-(3-dimethylaminopropyl)carbodiimide hydrochloride (EDC) and $N$-hydroxysulfosuccinimide sodium salt (sulfo-NHS) were purchased from Pierce (Rockford, IL). All of the reagents were of analytical grade or of the highest purity commercially available; they were used as received. All solutions were prepared with deionized water (Milli-Q, Bedford, MA) having a resistivity of no less than

(25) Welch, C. M.; Compton, R. G. Anal. Bioanal. Chem. 2006, 384, 601-619. (26) (a) Pena, J. M.; Allen, N. S.; Edge, M.; Liauw, C. M.; Noiset, O.; Valange, B. J. Mater. Sci. 2001, 36, 4419-4431. (b) Pena, J. M.; Allen, N. S.; Edge, M.; Liauw, C. M.; Valange, B. Polym. Degrad. Stab. 2001, 72, 259-270.

(27) (a) Liu, G.; Wang, J.; Kim, J.; Jan, M. R.; Collins, G. E. Anal. Chem. 2004, 76, 7126-7130. (b) Wang, J.; Liu, G.; Merkoci, A. J. Am. Chem. Soc. 2003, 125, 3214-3215. (c) Wang, J.; Liu, G.; Wu, H.; Lin, Y. Small 2008, 4, 8286.

(28) (a) Willner, I.; Patolsky, F.; Wasserman, J. Angew. Chem., Int. Ed. 2001, 40, 1861-1864. (b) Hansen, J. A.; Wang, J.; Kawde, A. N.; Xiang, Y.; Gothelf, K. V.; Collins, G. J. Am. Chem. Soc. 2006, 128, 2228-2229.

(29) (a) Liu, G.; Wang, J.; Wu, H.; Lin, Y.; Lin, Y. Electroanalysis 2007, 19, 777-785. (b) Joseph, W. Electroanalysis 2007, 19, 769-776. 
$18 \mathrm{M} \Omega \cdot \mathrm{cm}$. The CNPs were kindly provided by Dr. K. C. Hwang at National Tsing Hua University. ${ }^{30}$

Apparatus. Scanning electron microscopy (SEM) images of the SPGE surface were obtained using a JEOL JSM-7000F thermaltype field emission scanning electron microscope (Akishima, Tokyo, Japan). Transmission electron microscopy (TEM) images of the CdS NPs were obtained using a JEOL JEM-2100 highresolution transmission electron microscope (Akishima, Tokyo, Japan). Centrifugation was performed using a HERMLE $Z 36$ HK apparatus (Wehingen, Germany). Absorption spectra were acquired using a GBC UV-vis spectrophotometer (Dandenong, VIC, Australia). Fluorescence measurements were performed using a Varian Eclipse fluorescence spectrophotometer (Palo Alto, CA). Powder X-ray diffraction spectra were collected using an XRD7000 X-ray diffractometer (Chiyoda, Tokyo, Japan). All optical measurements were performed at room temperature. All $\mathrm{pH}$ measurements were performed using a Sartorius AG basic meter PB-10 (Göttingen, Germany). Time-of-flight secondary ion mass spectrometry (TOF-SIMS) data were acquired using a Cameca IMS-4f secondary ion mass spectrometer (Courbevoie, France). Typical analysis conditions involved a $25 \mathrm{keV}$ and a $1.5 \mathrm{pA}$ pulsed $\mathrm{Ga}^{+}$beam at a $45^{\circ}$ incidence, rastered over an area of $130 \mu \mathrm{m}$ $\times 130 \mu \mathrm{m}$. The ion fluence was maintained at less than $3 \times 10^{12}$ ions $/ \mathrm{cm}^{2}$ to ensure quasi-static conditions. X-ray photoelectron spectroscopy (XPS) analyses were conducted using an ULVACPHI XPS spectrometer (model PHI Quantera SXM, Chigasaki, Japan). Square wave voltammetric stripping measurements were performed using a $\mathrm{CH}$ Instruments $\mathrm{CHI} 1030$ electrochemical analyzer/workstation (Austin, TX). The disposable electrochemical SPGE, comprising a graphite working electrode, a carbon counter electrode, and a $\mathrm{Ag} / \mathrm{AgCl}$ reference electrode, was purchased from Zensor R\&D (Taichung, Taiwan).

Preparation of the CdS QDs. Many methods have been reported for the synthesis of CdS QDs, but most of them are costly, require extreme reaction conditions, or result in low product yields. ${ }^{31}$ In this study, MUA-capped CdS QDs were synthesized in aqueous solution at ambient temperature using a one-pot procedure. In short, MUA (40 mmol/L, $0.43 \mathrm{~g})$ was dissolved in Tris $(400 \mathrm{mmol} / \mathrm{L})$ and the $\mathrm{pH}$ adjusted to 8.5-9.0. $\mathrm{Cd}\left(\mathrm{NO}_{3}\right)_{2}(40 \mathrm{mmol} / \mathrm{L}, 25 \mathrm{~mL})$ was added dropwise into this solution, and then the mixture was stirred for $30 \mathrm{~min}$. Subsequently, $\mathrm{Na}_{2} \mathrm{~S}(1 \mathrm{mmol})$ dissolved in water $(25 \mathrm{~mL})$ was slowly added into the vortex of the above mixture to reach a $\mathrm{S} / \mathrm{Cd}$ molar ratio of 1:1. The final concentrations of $\mathrm{Cd}^{2+}$ and $\mathrm{S}^{2-}$ were both $10 \mathrm{mmol} / \mathrm{L}$. The mixture was stirred for $1 \mathrm{~h}$ at room temperature, and then isopropyl alcohol $(200 \mathrm{~mL})$ was added to the MUA-capped CdS solution. The bright-yellowish colloids were obtained after centrifugation at $4000 \mathrm{rpm}$ for 10 min. The resulting MUA-capped CdS QDs were characterized using X-ray diffractometry and TEM.

The CdS nanocrystal biotracer, $\alpha \mathrm{CEA}-\mathrm{CdS} \mathrm{QD}$, was synthesized based on a modification of the procedure reported by Wang

(30) Hsin, Y. L.; Lin, C. F.; Liang, Y. C.; Hwang, K. C.; Horng, J. C.; Ho, J. a. A.; Lin, C. C.; Hwu, J. R. Adv. Funct. Mater 2008, 18, 2048-2056.

(31) Peng, X.; Schlamp, M. C.; Kadavanich, A. V.; Alivisatos, A. P. J. Am. Chem. Soc. 1997, 119, 7019-7029. et al. ${ }^{32}$ The MUA-capped CdS QDs were first dissolved in phosphate-buffered saline (PBS) solution $(20 \mathrm{mM}, \mathrm{pH} 8.6)$ at a concentration of $2.88 \mathrm{mM}$. A portion $(10 \mu \mathrm{L})$ of this suspension was then diluted 10-fold with PBS, and then EDC $(1 \mathrm{mg})$ and sulfoNHS (1 mg) were added. The mixture was stirred for $30 \mathrm{~min}$, and then it was centrifuged (4000 rpm, $2 \mathrm{~min})$ to remove the supernatant. The anti-CEA antibody $(450 \mu \mathrm{g} / \mathrm{mL}, 0.85 \mathrm{~mL})$ was added to the functionalized CdS QD pellets, and then the mixture left to stand for $1 \mathrm{~h}$. Finally, the $\alpha \mathrm{CEA}-\mathrm{CdS}$ QD bioconjugates were collected through centrifugation (10 $000 \mathrm{rpm}, 5 \mathrm{~min})$ and then resuspended in Tris $-\mathrm{HCl}$ buffer $(50 \mathrm{mM}$ Tris $-\mathrm{HCl}$ containing $100 \mathrm{mM} \mathrm{NaCl}$ and $5 \mathrm{mM} \mathrm{KCl}$; $\mathrm{pH}$ 7.4; $1.2 \mathrm{~mL}$ ).

Fabrication of Immunosensors. The graphite working electrode of the SPGE was fabricated by placing a drop $(6 \mu \mathrm{L})$ of a suspension of CNP $(5 \mathrm{mg} / \mathrm{mL})$ and PEI $(3 \mathrm{mg} / \mathrm{mL})$ in $0.1 \mathrm{M}$ PBS $(\mathrm{pH} 8.66)$ onto the surface and leaving it to dry for $24 \mathrm{~h}$ at ambient temperature. Covalent immobilization of $\alpha \mathrm{CEA}$ on the CNP-PEI/SPGE was performed by placing a drop $(5 \mu \mathrm{L})$ of $2 \%$ glutaraldehyde (in 0.1 M PBS, pH 7.4) onto the surface, leaving it for $3 \mathrm{~h}$, and then placing a drop $(5 \mu \mathrm{L})$ of $\alpha \mathrm{CEA}$ solution (50 $\mu \mathrm{g} / \mathrm{mL}$ in $0.1 \mathrm{M}$ PBS, $\mathrm{pH} 7.0$ ) on the SPGE surface for $30 \mathrm{~min}$ before washing slowly with a stream of $0.1 \mathrm{M}$ PBS (pH 7.0).

Assay Procedure. The $\alpha$ CEA-immobilized CNP-PEI/SPGE was incubated for 20 min with the CEA standard in Tris $-\mathrm{HCl}$ buffer $(5 \mu \mathrm{L})$ and then washed with Tris- $\mathrm{HCl}$ buffer. The CNP-PEI/SPGE was then incubated for 15 min with a 5 -folddiluted solution of the $\alpha \mathrm{CEA}-\mathrm{CdS}$ QDs in Tris- $\mathrm{HCl}$ buffer ( 5 $\mu \mathrm{L})$. The SPGE was washed again with Tris $-\mathrm{HCl}$ buffer to remove any unbound $\alpha \mathrm{CEA}-\mathrm{CdS}$ QD. Dissolution of the bound CdS QD was performed through the sequential addition of $\mathrm{HNO}_{3}(0.1 \mathrm{M}$, $5 \mu \mathrm{L})$ and acetate buffer $(0.1 \mathrm{M}, \mathrm{pH} 4.6,40 \mu \mathrm{L})$ spiked with mercury(II) nitrate $(65 \mathrm{ppm})$ to enhance the metal deposition and stripping performance. The electrochemical stripping detection process involved pretreatment at $+0.6 \mathrm{~V}$ for $1 \mathrm{~min}$, electrodeposition at $-1.0 \mathrm{~V}$ for $1 \mathrm{~min}$, and stripping from -1.0 to $-0.1 \mathrm{~V}$ using a square wave voltammetric waveform (potential steps, $4 \mathrm{mV}$; frequency, $15 \mathrm{~Hz}$; amplitude, $25 \mathrm{mV}$ ).

\section{RESULTS AND DISCUSSION}

Characterization of CdS QDs and $\alpha \mathrm{CEA}-\mathrm{CdS}$ QD Biotracers. We used TEM to study the morphology of the MUA-capped CdS QDs. The typical TEM image in Figure 1A reveals that these CdS QDs were irregular spherical nanocrystals having diameters of ca. $3 \mathrm{~nm}$. The powder X-ray diffraction (XRD) pattern of the prepared CdS QDs (Figure 1B) displays only two broad peaks, centered at values of $2 \theta$ of $28^{\circ}$ and $48^{\circ}$, similar to the results reported by Chen et al..$^{33}$ These peaks were broadened considerably as a result of the quantum size effect of the nanocrystallites. We used the Debye-Scherrer formula

$$
D=0.9 \lambda / \beta \cos \theta
$$

where $D$ is the diameter of the nanocluster, $\lambda$ is the wavelength of the incident X-rays, $\beta$ is the full width at half-maximum, and $\theta$ is the diffraction angle, to calculate an average crystal size of 1.1

(32) Wang, S.; Mamedova, N.; Kotov, N. A.; Chen, W.; Studer, J. Nano Lett. 2002, 2, 817-822.

(33) Chen, J.; Zheng, A. F.; Gao, Y.; He, C.; Wu, G.; Chen, Y.; Kai, X.; Zhu, C. Spectrochim. Acta, Part A 2008, 69, 1044-1052. 
(A)

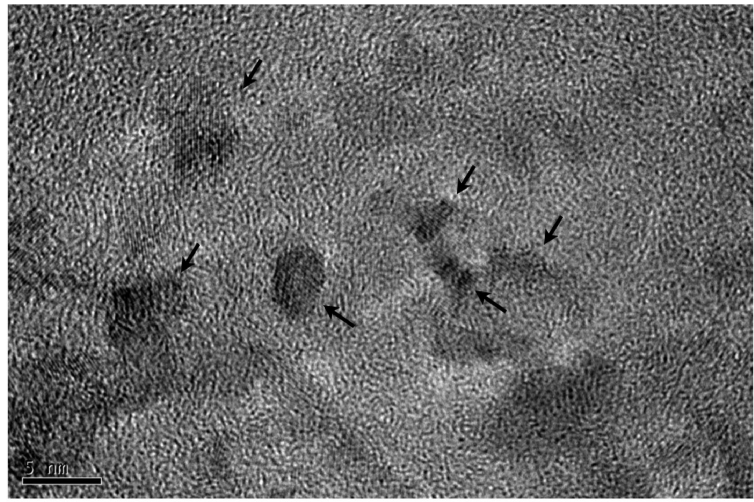

(B)
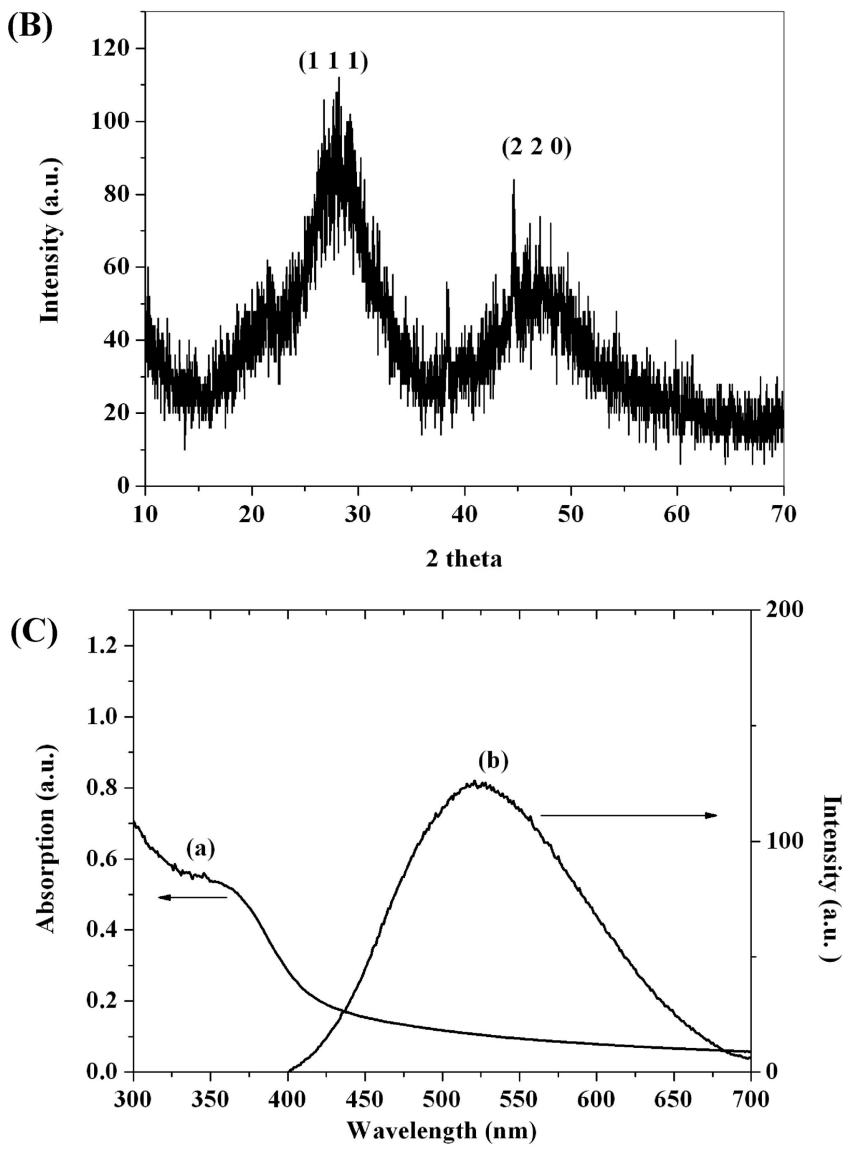

Figure 1. (A) TEM image, (B) XRD spectrum, and (C) absorption (a) and fluorescence (b) spectra $\left(\lambda_{\mathrm{em}} / \lambda_{\mathrm{ex}}=525 / 370 \mathrm{~nm}\right)$ of the MUAcapped CdS QDs.

$\mathrm{nm}$ for the CdS QDs. ${ }^{33}$ This value is slightly smaller than that determined from TEM visualization, possibly because of the aggregated structure of the CdS QDs under TEM analysis, due to static attraction of the surface groups. Figure $1 \mathrm{C}$ displays the fluorescence and absorption spectra. The excitation spectrum of the CdS QDs synthesized in this study displays a broad and symmetrical emission ranging from 400 to $700 \mathrm{~nm}$, indicating the potential for high-energy electronic transitions. In addition, an absorption band appears at ca. $360 \mathrm{~nm}$.

We determined the coupling efficiency of $\alpha \mathrm{CEA}$ onto CdS QDs by using the following expression: (amount of anti-CEA coupled/ amount of anti-CEA offered) $\times 100 \%$. We calculated the amount of anti-CEA coupled by subtracting the total amount of anti-CEA found in the wash fraction from the amount of anti-CEA offered. The coupling efficiency was determined by measuring the absor- bance at $595 \mathrm{~nm}\left(A_{595}\right)$ using a Bio-Rad protein assay kit. We obtained a coupling efficiency of ca. 35\%, meaning that ca. 135 $\mu \mathrm{g}$ of $\alpha \mathrm{CEA}$ was successfully conjugated to ca. $2.5 \times 10^{-3} \mu \mathrm{mol}$ of the CdS QDs. Thus, ca. 3 CdS QDs were conjugated to one antibody molecule.

Fabrication of Carbon Nanomaterial-Modified SPGE. Scheme 1 presents a schematic representation of the analytical technique developed in this study for the amplified detection of CEA. Surface-modified electrode fabrication technologies are commonly used to increase the performance of an electrode toward an analyte. Carbon nanomaterials, such as CNTs, generally do not disperse readily in liquid or polymer matrixes because the extensive $\pi$-electron system present on the tube walls leads to enhanced van der Waals forces. The resulting side-by-side aggregates of CNTs, so-called "ropes" ${ }^{34}$ are very difficult to disrupt. Hence, to prevent the CNPs from aggregating into clumps, we enclosed them in a polymeric matrix. When we coated a PEI layer over the surface of the CNPs, the positively charged PEI acted as a glue to bridge the neighboring NPs. We used the amino groups present in the polymer chains to immobilize the antibody. The surface morphology of the modified SPGE is an important factor affecting the performance of the immunosensor. Figure 2 displays SEM images of the morphologies of CNP-PEI/SPGE. The surface of the modified SPGE possessed a porous structure that resulted from the presence of the thin film of the CNP-PEI nanocomposite. The performance of our new CNP-PEI/SPGE was demonstrated in connection with the detection of free $\mathrm{Cd}^{2+}$ ions in solution. Figure 3 compares the square wave anodic stripping voltammetry (SWASV) responses of equal amount of CdS QDs on SPGE and CNP-PEI/SPGE. Both electrodes give one well-defined oxidation peaks at ca. $-0.84 \mathrm{~V}$ for the $\mathrm{Cd}^{2+}$ ions in an acetate buffer containing $65 \mathrm{ppm} \mathrm{Hg}^{2+}$. As expected, the CNP-PEI/SPGE provided a more favorable voltammetric response relative to those of SPGE and the PEI-modified SPGE. Thus, CNP-PEI/SPGE films enhanced the peak currents because of their unique electrical properties.

Antibody Immobilization. In an immunoanalytical system, the immobilization of biomolecules is fundamentally important for the successful development of an immunosensor. At present, the most common immobilization methods are based on adsorption, sandwich entrapment, covalent bonding, and cross-linking techniques. A basic limitation of passive trapping or adsorption is the instability of immobilized proteins during continuous use. A major limitation of the entrapment technique is the additional diffusion barrier resulting from the entrapped materials; this problem can be minimized by increasing the porosity of the matrix. One of the problems frequently associated with covalent bonding is the decrease in protein bioactivity when the proteins are exposed to reactive groups or harsh reaction conditions during the coupling reaction.

In this study, we immobilized $\alpha \mathrm{CEA}$ molecules onto the CNP-PEI/SPGE using glutaraldehyde, a common homobifunctional cross-linker. In conventional polymer-protein immobilization, the protein molecules usually reside in the bulk, thereby

(34) (a) Cowley, J. M.; Sundell, F. A. Ultramicroscopy 1997, 68, 1-12. (b) Monthioux, M.; Smith, B. W.; Burteaux, B.; Claye, A.; Fischer, J. E.; Luzzi, D. E. Carbon 2001, 39, 1251-1272. (c) Rinzler, A. G.; Liu, J.; Dai, H.; Nikolaev, P.; Huffman, C. B.; Rodriguez-Macias, F. J.; Boul, P. J.; Lu, A. H.; Heymann, D.; Colbert, D. T. Appl. Phys. A 1998, 67, $29-37$. 


\section{Scheme 1. Cartoon Representation of the Assembly and Amplified Detection of CEA Using the aCEA-CdS Biotracer and the CNP-PEI/SPGE}

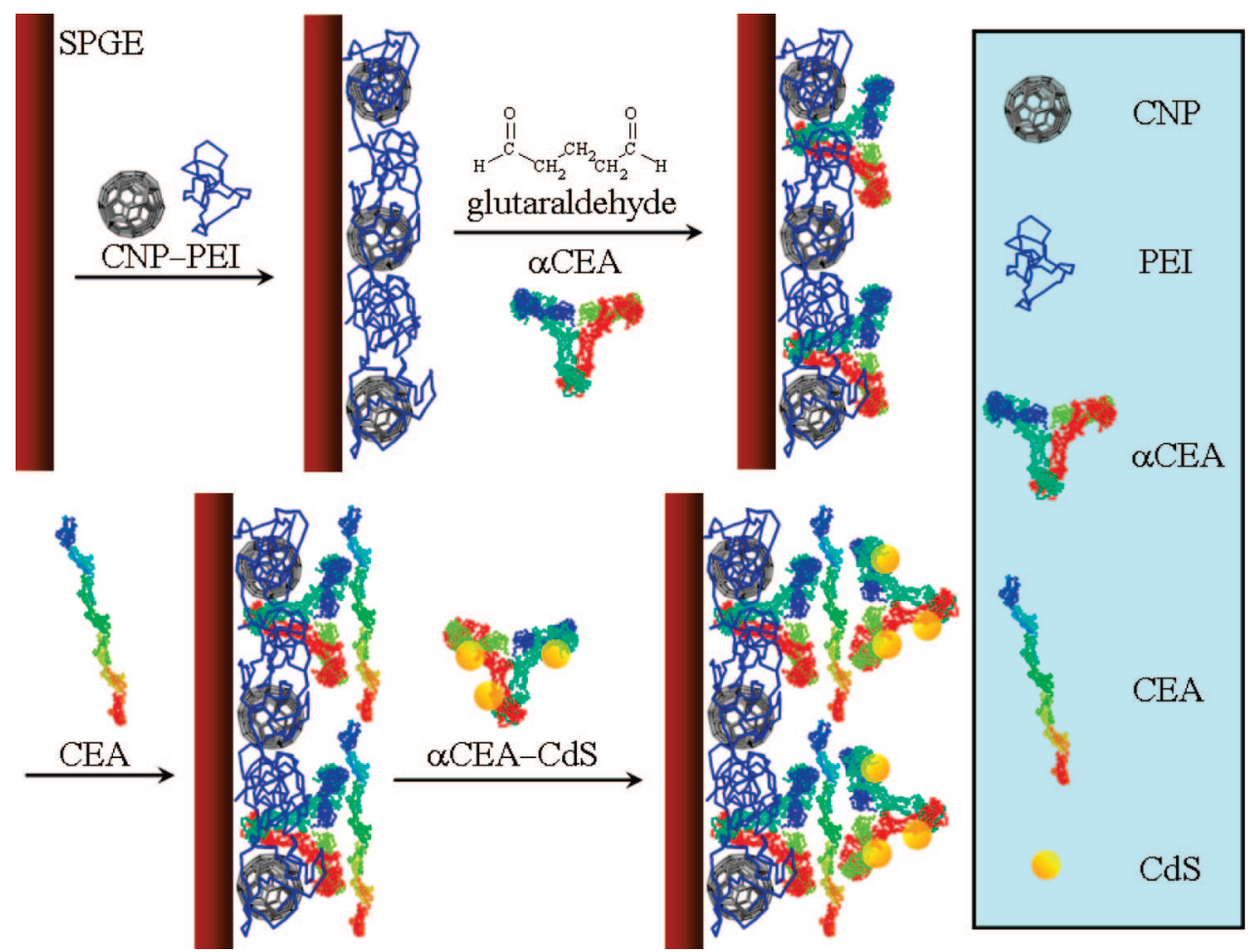

creating steric hindrance with regard to the accessibility of either the antigen or antibody toward the entrapped counter immunological moiety. In contrast, covalent immobilization of $\alpha \mathrm{CEA}$ units on the CNP-PEI/SPGE surface could occur only on the top layers. Hence, this method occurs with no loss of the biological activity or the content of antibodies during the washing steps. The higher surface area of the CNP (diameter: 50-100 nm)modified electrode allowed more antibodies to be accommodated on the electrode. The resulting surface presenting antibody molecules was readily detected through XPS analysis. The antibody-immobilized CNP-PEI/SPGE (Figure 4A) exhibited a signal at $288.7 \mathrm{eV}$, indicating the presence of $\mathrm{CO}_{2} \mathrm{H}$ units at the surface, in addition to signals representing $\mathrm{C}-\mathrm{C}(285.4 \mathrm{eV})$ and $\mathrm{C}-\mathrm{N}(286.6 \mathrm{eV})$ groups. We detected no such signal for $\mathrm{CO}_{2} \mathrm{H}$ groups on the bare CNP-PEI/SPGE (Figure 4B), confirming the successful deposition of anti-CEA antibody mol- ecules on the surface of the SPGE. We performed SIMS analysis using a TOF-SIMS instrument equipped with a gallium analysis ion source to confirm the successful deposition of the $\alpha \mathrm{CEA}$ molecules. We identified three species, $\mathrm{C}_{8} \mathrm{H}_{10} \mathrm{~N}^{+}, \mathrm{C}_{9} \mathrm{H}_{8} \mathrm{~N}^{+}$, and $\mathrm{C}_{8} \mathrm{H}_{10} \mathrm{NO}^{+}$, in the TOF-SIMS spectra on our $\alpha \mathrm{CEA}$-immobilized SPGEs (Figure 5) that were related only to the protein; these signals represent phenylalanine, tryptophan, and tyrosine, respectively. ${ }^{35}$

We incubated the $\alpha \mathrm{CEA}$ immobilized on the electrode with a solution of CEA $(5 \mu \mathrm{L})$ for various periods of time. The intensity of the SWASV signal increased rapidly upon increasing the incubation time to $20 \mathrm{~min}$, but no further significant increases occurred upon increasing the incubation time any further. Thus, the optimal incubation time was $20 \mathrm{~min}$. The association of the CEA units with the sensing interface was then amplified by the binding of the $\alpha \mathrm{CEA}-\mathrm{CdS}$ QD biolabels for $20 \mathrm{~min}$. The content

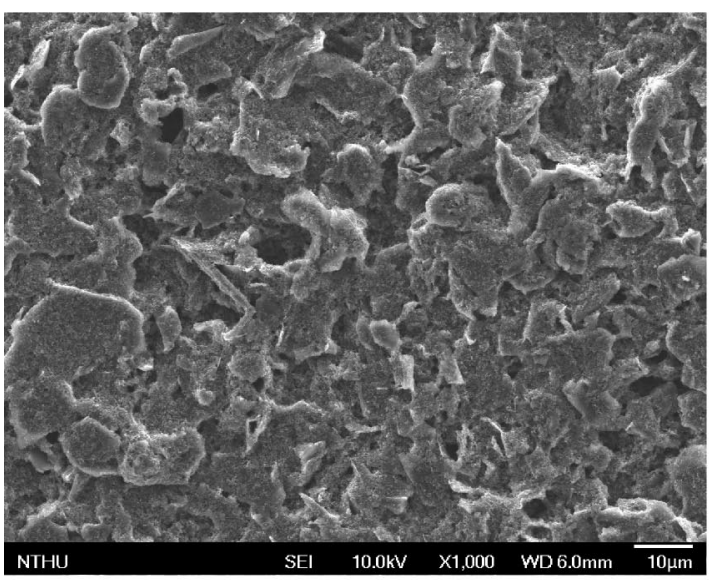

Bare SPGE

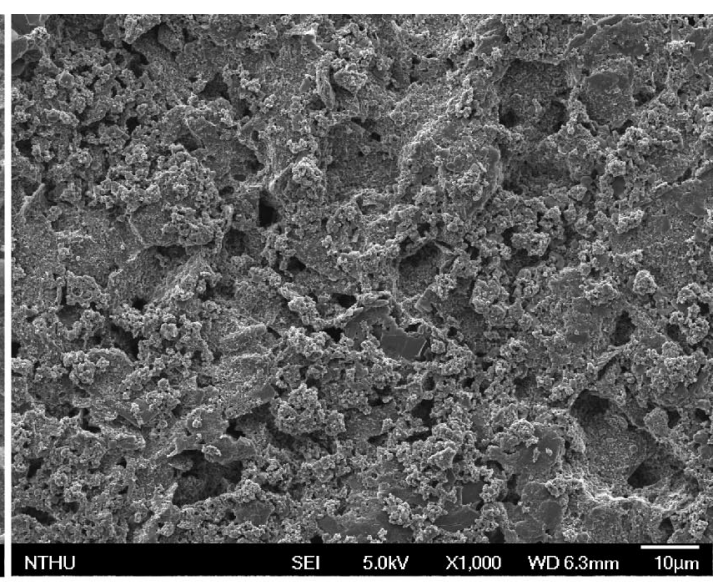

CNP-PEI/SPGE

Figure 2. SEM images of the bare SPGE and the CNP-PEI-modified SPGE. 


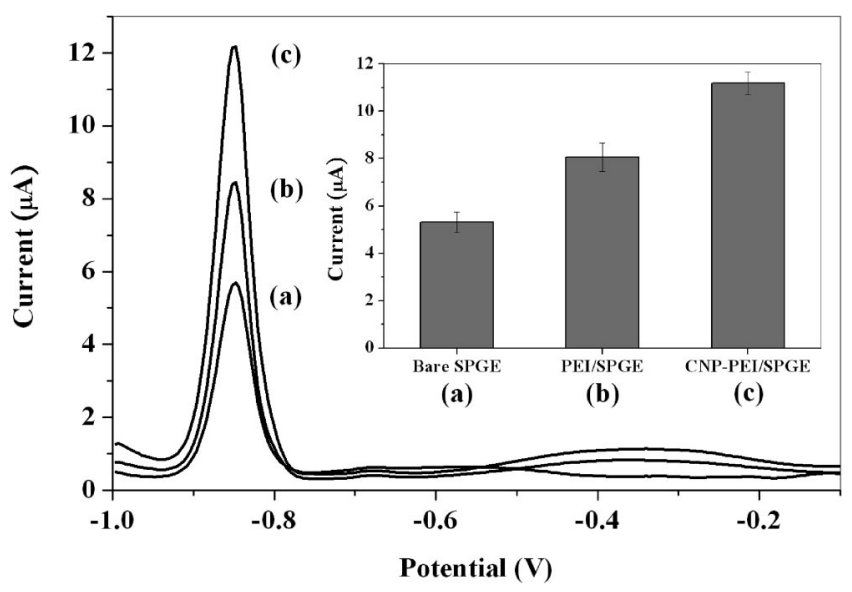

Figure 3. SWASV analysis of CdS QDs using the (a) bare SPGE, (b) PEI/SPGE, and (c) CNP-PEI/SPGE. The electrochemical stripping detection process involved pretreatment at $+0.6 \mathrm{~V}$ for $1 \mathrm{~min}$, electrodeposition at $-1.0 \mathrm{~V}$ for $1 \mathrm{~min}$, and stripping from -1.0 to -0.1 $\mathrm{V}$ using a square wave voltammetric waveform (potential steps, 4 $\mathrm{mV}$; frequency, $15 \mathrm{~Hz}$; amplitude, $25 \mathrm{mV}$ ).
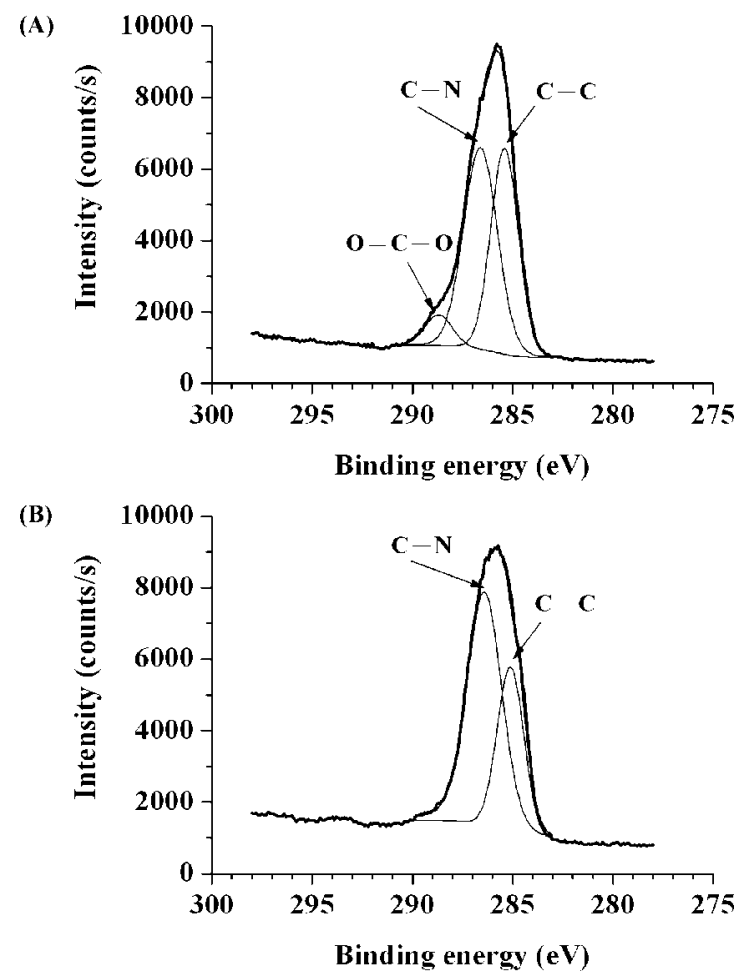

Figure 4. X-ray photoelectron spectra of the surfaces of the $(A)$ antibody-deposited CNP-PEI/SPGE $(50 \mu \mathrm{g} / \mathrm{mL} \alpha$ CEA used) and the (B) CNP-PEI/SPGE.

of bound $\alpha \mathrm{CEA}-\mathrm{CdS} \mathrm{QD}$ biotracers on the electrode surface was proportional to the amount of CEA present in the sample.

Our new electrochemical immunosensing technique involved the binding of secondary antibodies linked to the CdS QD (forming biotracers) and the $\alpha \mathrm{CEA}$-sensitized electrode surface, which was sensitive to the concentration of CEA. The method we chose to detect the bound CdS QD biotracers was SWASV, which combines the amplification of stripping voltammetry with the ultrasensitivity and low background noise of square wave scanning. The coupling of the sensitive CNP-PEI/SPGE- and $\alpha \mathrm{CEA}-\mathrm{CdS} \mathrm{QD}-$ based stripping assays provided good perfor-

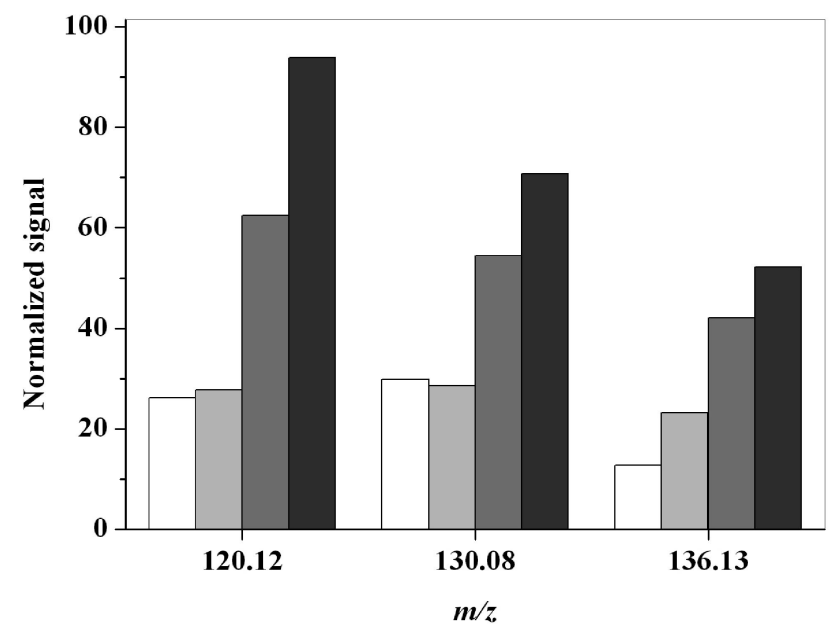

Figure 5. Characteristic TOF-SIMS fragments $\left(\mathrm{C}_{8} \mathrm{H}_{10} \mathrm{~N}^{+}, \mathrm{C}_{9} \mathrm{H}_{8} \mathrm{~N}^{+}\right.$, and $\mathrm{C}_{8} \mathrm{H}_{10} \mathrm{NO}^{+}$) detected from the immobilized $\alpha \mathrm{CEA}$ : white, bare SPGE; light gray, CNP-PEI/SPGE; dark gray, $50 \mu \mathrm{g} / \mathrm{mL} \alpha \mathrm{CEA}$ immobilized CNP-PEI/SPGE; black, $1 \mathrm{mg} / \mathrm{mL} \alpha C E A$-immobilized CNP-PEI/SPGE.

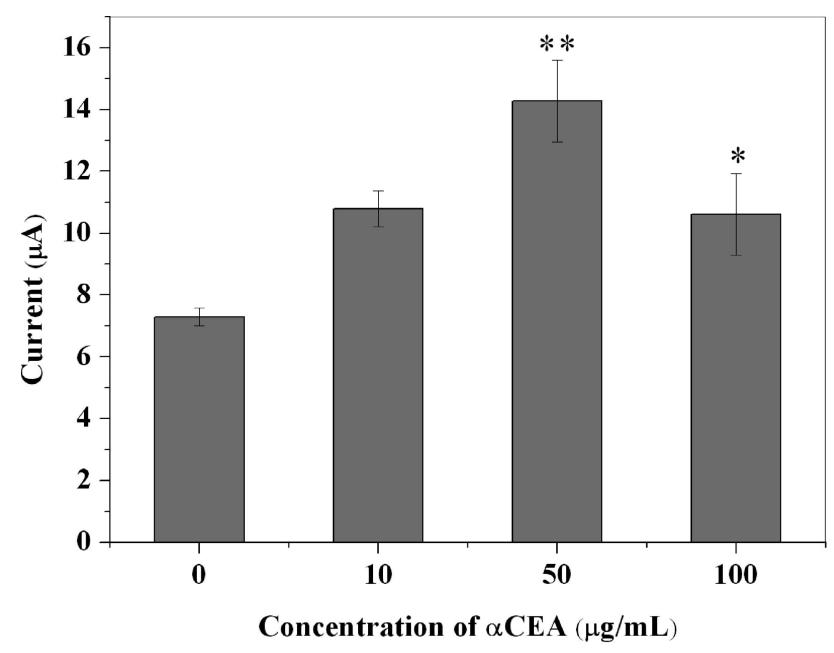

Figure 6. Effect of the concentration of $\alpha C E A$ on its immobilization. (**, $p<0.01$ as compared with the absence of $\alpha \mathrm{CEA} ; *, p<0.05$ as compared with the absence of $\alpha \mathrm{CEA}$.)

mance. In these cases, the optimum amount of antibody used for immobilization on the SPGE was investigated; and results are shown in Figure 6. Several SPGEs bearing different amounts of antibody were fabricated and tested for the same concentration of target analyte, CEA $(1 \mathrm{ng} / \mathrm{mL})$. It is clear from Figure 6 that the sensor response increased significantly $(p<0.01)$ when the concentration of antibody loaded on the electrode surface was varied from 0 to $50 \mu \mathrm{g} / \mathrm{mL}$. Upon increasing the antibody concentration further to $100 \mu \mathrm{g} / \mathrm{mL}$, no signal enhancement was observed. Hence, we found that the optimal concentration was $50 \mu \mathrm{g} / \mathrm{mL}$. The coupling efficiency of ca. $30 \%$ of the applied antiCEA antibody, determined using the Bio-Rad protein assay kit, suggested that each SPGE bore $75 \mathrm{ng}$ of $\alpha \mathrm{CEA}$ on the working electrode.

Analytical Calibration. The analytical calibration (as shown in Figure 7) was performed-using various concentrations of the target analyte- to determine the sensitivity of the immunosensor

(35) Azioune, A.; Pireaux, J. J.; Houssiau, L. Appl. Surf. Sci. 2004, 231-232, 402-405. 


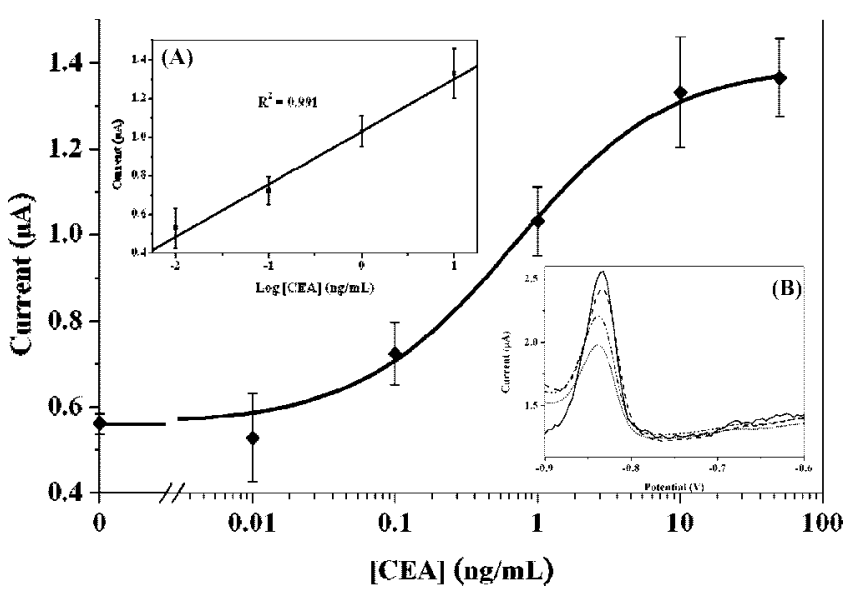

Figure 7. Dose-response curves obtained from the $\alpha \mathrm{CEA}$-immobilized CNP-PEI/SPGE. Each point represents the mean of three measurements; error bars: \pm 1 SD. The insets display $(A)$ the linear fit and $(B)$ square wave anodic stripping voltammograms of the central data.

Table 1. Recovery of CEA from Urine Samples
\begin{tabular}{cccc}
\hline & $\mathrm{CEA}, \mathrm{ng} / \mathrm{mL}(n=5)$ & \\
\cline { 2 - 3 } sample matrix & added & found & recovery $\%$ \\
$1 / 20 \times$ human urine & 1.000 & 1.088 & 108.8 \\
\hline
\end{tabular}

\section{Table 2. Comparison of Detection Limit and Linear Range in CEA Analysis}

method

piezoelectric immunosensor iridium oxide film-enhanced impedance immunosensor

electrochemical immunosensor modified by gold nanoparticles and chitosan

electrochemical immunosensor by phenylboronic acid self-assembly layer

$\mathrm{DAB}-\mathrm{H}_{2} \mathrm{O}_{2}-\mathrm{HRP}$ voltammetric enzyme-linked immunoassay

electrochemical immunosensor using thionine-doped magnetic gold nanospheres and horseradish

carbon nanoparticle-enhanced immunoelectrochemical detection (present study) free of CEA under identical conditions-when using this electrochemical immunosensor was $32 \mathrm{pg} / \mathrm{mL}$ (equivalent to $160 \mathrm{fg}$ of CEA in a $5 \mu \mathrm{L}$ sample), with a $99.7 \%$ level of confidence. Replicated analysis of various amounts of CEA led to an average coefficient variation $(\mathrm{CV} \%)$ of no more than $10 \%$, indicating that the reproducibility of the immunosensor's response and the fabrication procedure were acceptable.

Urine Sample Analysis. Determination of urinary CEA levels is very important for the early detection of urothelial carcinoma. We spiked human urine samples from laboratory personnel with CEA and analyzed them to determine the effects of a real matrix. We measured the stability of the analysis of each urine sample by performing five consecutive experiments with the same human sample over a period of ca. $1 \mathrm{~h}$. We obtained a CV\% of $17 \%$ for the peak current from the five replicates. The experimental recovery was acceptable (Table 1).

\section{CONCLUSION}

This paper presents a novel, yet simple, electrochemical immunosensing platform for the detection of the cancer marker CEA. We prepared the immunosensor by immobilizing $\alpha$ CEA on an SPGE nanocomposite incorporating PEI-coated CNPs. The integration of the CNP-PEI/SPGE and the $\alpha \mathrm{CEA}-\mathrm{CdS}$ QDs improved the electrochemical immunoassay detection level. The presence of the CNPs improved the performance of the electrochemical reaction of the substrate and increased the sensitivity of the immunosensor for the diagnosis of CEA. This technique had adequate precision and sensitivity, with an LOD of $160 \mathrm{fg}$ of CEA (equivalent to $5 \mu \mathrm{L}$ of a $3.2 \times 10^{-11} \mathrm{~g} / \mathrm{mL}$ solution) and an acceptable dynamic range of 3 orders of magnitude. In addition, only $5 \mu \mathrm{L}$ of sample was required to perform one single assay. Comparison of detection limit and linear range of various methods in analysis of CEA is listed in Table 2. The sensitivity of current electrochemical immunosensing system for CEA is comparable to those of previously reported electrochemical systems. ${ }^{13 \mathrm{c}}$ The immunosensor exhibited satisfactory reproducibility for the analysis of human urine samples. We believe that this single-use, disposable SPGE-based electrochemical immunosensor has potential for further development into practical clinic cancer diagnosis systems, e.g., point-of-care or home-care diagnostics.

\section{ACKNOWLEDGMENT}

We thank the National Science Council of Taiwan for financial support under Grants NSC 95-2113-M-007-040-MY3, 96-2627-B-007013, 96-2120-M-002-007, and National Tsing Hua University under Boost Program Grant. Yeh-Chun Lin and Li-Sheng Wang contributed equally to this publication.

Received for review September 1, 2008. Accepted December 29, 2008.

\section{$\mathrm{AC} 801832 \mathrm{H}$}

(36) Ding, Y.-j.; Wang, H.; Jiang, J.-h.; Shen, G.-l.; Yu, R.-q. Chin. J. Chem. 2007, $25,1288-1293$

(37) Lin, J.; Qu, W.; Zhang, S. Anal. Sci. 2007, 23, 1059-1063.

(38) Zhang, X.-t.; Wu, Y.-f.; Tu, Y.-f.; Liu, S.-q. Analyst 2008, 133, 485-492.

(39) Zhang, S.; Yang, J.; Lin, J. Bioelectrochemistry 2008, 72, 47-52. 